VIII ${ }^{\text {èmes }}$ Journées Nationales Génie Civil - Génie Côtier, Compiègne, 7-9 septembre 2004

\title{
Comportement d'éprouvettes de béton et de mortier à la mer
}

\author{
Zhibo Duan, Daniel Levacher, Zhenzhong Tang \\ Université de Caen, Centre de géomorphologie, GRGC \\ 24 rue des Tilleuls, 14000 Caen, France
}

\begin{abstract}
Résumé
Initié il y a 30 ans, un important programme de recherche relatif au comportement mécanique et physico-chimique de bétons et de mortiers à la mer, a permis d'acquérir de nombreux résultats d'essais à différentes périodes définies contractuellement [1]. Différents ciments avaient été sélectionnés pour des contenus variables en clinker et liants hydrauliques, et différentes teneurs en aluminates tricalciques et sulfates, composés pouvant réagir avec l'eau de mer. Le paramètre $\mathrm{E} / \mathrm{C}$ qui contribue de manière très sensible à la résistance et à la pénétration des ions chlorure a été maintenu constant et voisin de 0.5 pour tous les dosages des bétons étudiés. L'article traite de l'analyse de résultats d'essais de pénétration des ions chlorure effectués dans une section d'éprouvette de béton $20 \times 20 \times 80 \mathrm{~cm}^{3}$. Sont rappelées les tendances évolutives des résistances mécaniques de ces mêmes bétons. Les mesures de pénétration des ions chlorure sont présentées en relation avec la catégorie de ciment et les dosages retenus pour les bétons testés.
\end{abstract}

\begin{abstract}
$\underline{\text { Abstract }}$
Long-term effect on the mortar and concrete in marine environment has been a subject of numerous studies. The effect of sea-water on the mechanical and physico-chemical properties of mortar and concrete exposed for different time span has been analysed in this paper. Different types of cements had been chosen with different levels of clinker and hydraulic binders as well as different amounts of aluminates and sulfates which enhance the chemical reaction with sea water. E/C which contribute appreciably to UC strength and chloride penetration, was kept constant at 0.5 for all the concretes. This paper analyses the results on the chloride ion penetration at a section of concrete samples of dimension $20 \times 20 \times 80 \mathrm{~cm}$. The tendency evolutive of mechanical strength has been recalled. The tests results presented here show the relationship between the penetration of chloride ions with that of cement type and amount.
\end{abstract}

Mots-clés: Béton, mortier, eau de mer, résistance à la compression, pénétration des chlorures

Keywords: Concrete, mortar, sea water, UC strength, chloride penetration

\section{Introduction}

La présente étude a été menée à la suite du programme expérimental relatif à la durabilité des bétons et mortiers à la mer, élaboré en 1974 par la COmmission Permanente des Liants hydrauliques et des Adjuvants du béton -COPLA-, [1]. Elle avait pour objectif de suivre l'évolution dans le temps des résistances mécaniques et de faire état à 30 ans de la répartition des ions chlorure d'éprouvettes de béton immergées en zone de marnage au sein du port du Havre. Ces éprouvettes de béton sont soit des cylindres $16 \times 32 \mathrm{~cm}^{2}$, soit des poutres parallélépipédiques $20 \times 20 \times 80 \mathrm{~cm}^{3}$. 
Elles ont été confectionnées avec des ciments d'origine différente et ce, pour plusieurs dosages. Le paramètre $\mathrm{E} / \mathrm{C}$ a été volontairement maintenu constant et voisin de 0.5 . Les tendances évolutives des résistances mécaniques sur une période de 30 ans sont d'abord discutées en fonction des catégories de ciments retenus et des essais de compression non confinée réalisés sur des cylindres et des cubes $20 \times 20 \times 20 \mathrm{~cm}^{3}$ issus des poutres de béton. Puis est établi à l'âge de 30 ans, la répartition des ions chlorure dans une section transversale de ces mêmes poutres. Les mesures de pénétration des ions chlorure ont ainsi permis de mettre en évidence, pour différentes catégories de ciment, une dissymétrie de la répartition. S'ensuit, une présentation des résultats en relation avec la catégorie de ciment considérée.

\section{$\underline{\text { 2.Catégories de ciment }}$}

Pour l'ensemble du programme COPLA [1],[2], 14 types de ciment ont été retenus. Leurs teneurs en $\mathrm{C}_{3} \mathrm{~A}, \mathrm{SO}_{3}, \mathrm{C}_{3} \mathrm{~S}$ et laitiers respectives sont rappelées au tableau 1.

Tableau 1: Les différents ciments utilisés dans le programme COPLA

\begin{tabular}{|l|ll|l|l|l|l|l|l|l|}
\hline Catégorie & $\begin{array}{l}\text { Marque } \\
\text { origine }\end{array}$ & $\begin{array}{l}\text { ou } \\
\text { Mortier } \\
\text { date) }\end{array}$ & $\begin{array}{l}\text { Béton } \\
\text { (date) }\end{array}$ & $\mathrm{C}_{3} \mathrm{~A}$ & $\mathrm{SO}_{3}$ & $\mathrm{C}_{3} \mathrm{~S}$ & $\begin{array}{l}\mathrm{C}_{3} \mathrm{~A}+ \\
0.27 \mathrm{C}_{3} \mathrm{~S}\end{array}$ & Laitiers \\
\hline $1:$ CPA400 & HTS & $25 / 02 / 74$ & $08 / 04 / 74$ & 3.84 & 1.87 & 70.53 & 22.88 & -- \\
\hline 1 bis $:$ CPA325 & HRC & $04 / 06 / 74$ & $02 / 09 / 74$ & 1.37 & 2.33 & 56.90 & 16.73 & -- \\
\hline $2:$ CPA325 & Chiron & $18 / 02 / 74$ & $28 / 05 / 74$ & 8.03 & 2.57 & 58.66 & 23.86 & -- \\
\hline $3:$ CPA325 & Lorraine & $17 / 04 / 74$ & $09 / 09 / 74$ & 11.27 & 3.25 & 37.91 & 21.51 & -- \\
\hline $4:$ CPA400 & Loire & $01 / 07 / 74$ & $09 / 09 / / 74$ & 7.42 & 3.40 & 63.92 & 24.67 & -- \\
\hline $5:$ CPA400 & $\begin{array}{l}\text { Clinker } \\
\text { Barilin }\end{array}$ & de & $11 / 09 / 74$ & $21 / 10 / 74$ & 7.62 & 4.46 & 54.23 & 22.26 & -- \\
\hline $6:$ CPA400 & $\begin{array}{l}\text { Ciments } \\
\text { Français }\end{array}$ & $12 / 03 / 74$ & $22 / 04 / 74$ & 14.66 & 2.93 & 43.99 & 26.54 & - \\
\hline $7:$ CPA325 & Vicat & $03 / 09 / 74$ & $16 / 09 / 74$ & 9.47 & 2.48 & 56.11 & 24.62 & -- \\
\hline $8:$ CPAL325 & $\begin{array}{l}\text { Ciments } \\
\text { Français }\end{array}$ & $10 / 04 / 74$ & $29 / 04 / 74$ & 17.30 & 2.50 & -- & & $20 \%$ \\
\hline $9:$ CPF325 & $\begin{array}{l}\text { Clinker } \\
\text { Barlin }\end{array}$ & de & $17 / 09 / 74$ & $29 / 10 / 74$ & -- & 3.80 & -- & -- & $30 \%$ \\
\hline $10:$ CPF325 & Vicat & $03 / 10 / 74$ & $18 / 11 / 74$ & -- & 3.17 & -- & -- & $30 \%$ \\
\hline $11:$ CLK325 & Lorraine & $23 / 04 / 74$ & $24 / 06 / 74$ & -- & 3.30 & -- & -- & -- \\
\hline $12:$ Prompt & Vicat & $28 / 06 / 76$ & $22 / 06 / 76$ & -- & -- & -- & -- & -- \\
\hline $\begin{array}{l}13 \\
\text { CPAZ400 }\end{array}$ & Lafarge & $13 / 12 / 78$ & $20 / 12 / 78$ & -- & - & - & -- & -- \\
\hline
\end{tabular}

\section{Conditions d'immersion des éprouvettes en mer}

C'est dans une partie du port de Havre en zone de marnage que les éprouvettes $20 \times 20 \times 80 \mathrm{~cm}^{3}$ ont été stockées en bas d'un mur de quai. Elles subissent l'effet des marées. Pour parer à ces dernières, des cases ont été construites de manière à éviter tout déplacement d'éprouvettes à l'aide de barreaux d'acier scellés dans les murets en parpaings. Ceci peut être observé sur la figure 1b. Dans cette configuration, chaque face ne subit pas les cycles mouillage-séchage et l'attaque des vagues de la même manière. Un repérage des faces a été opéré au moment de leur enlèvement. 


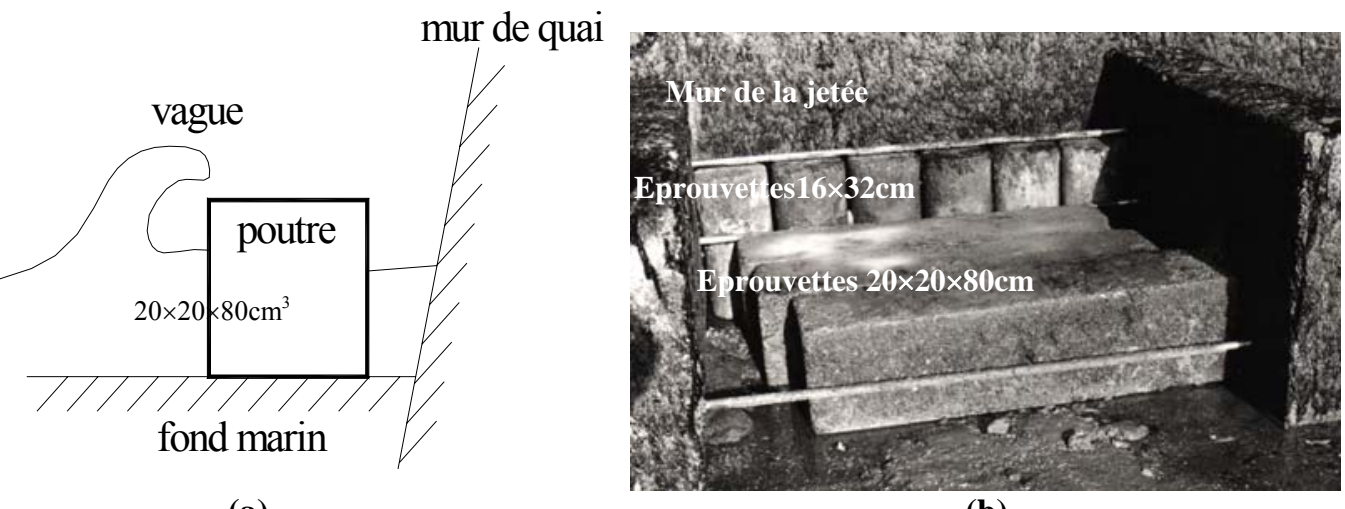

(a)

(b)

Figure 1 : Conservation et conditions d'exposition des éprouvettes à la vague

\section{Eprouvettes hors norme en 2004}

Les éprouvettes prismatiques (Norme NF P 18-401, [3]) de béton étaient constituées de prismes à section carrée de coté a et de longueur L. Elles étaient destinées à des essais de rupture par flexion en 4 points et de compression simple sur cubes dont les caractéristiques géométriques étaient définies selon la taille du plus gros granulat D (Norme NF P 18-402). Des éprouvettes prismatiques de béton ont donc été confectionnées aux dimensions indiquées dans le tableau 2 car la valeur de D retenue dans le programme COPLA n'excédait pas 50mm.

Tableau 2: Dimensions des éprouvettes prismatiques et cubiques

\begin{tabular}{|l|l|l|l|l|}
\hline \multicolumn{2}{|l|}{$\begin{array}{l}\text { Eprouvette } \\
\text { prismatique }\end{array}$} & \multicolumn{2}{|l|}{$\begin{array}{l}\text { Eprouvette } \\
\text { cubique }\end{array}$} & $\begin{array}{l}\mathrm{D} \\
(\mathrm{mm})\end{array}$ \\
\hline $\mathrm{a}(\mathrm{mm})$ & $\mathrm{L}(\mathrm{mm})$ & $\begin{array}{l}\text { Arête a Section } \\
(\mathrm{mm})\end{array}$ & $\begin{array}{l}\text { Section } \\
\left(\mathrm{cm}^{2}\right)\end{array}$ & $\leq 50$ \\
\hline 200 & 800 & 200 & 400 & \\
\hline
\end{tabular}

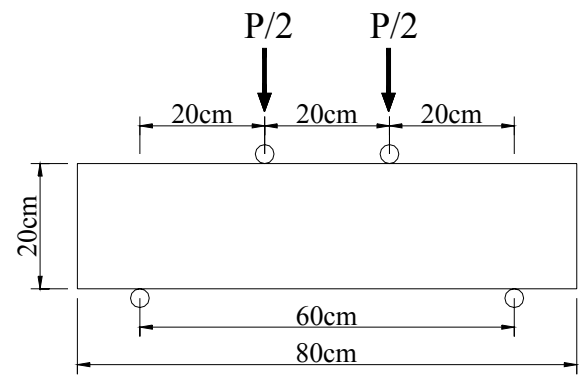

Figure 2 : Schéma d'un essai de flexion 


\section{Résistance à la compression simple}

Chaque éprouvette prismatique ou poutre en béton de $20 \times 20 \times 80 \mathrm{~cm}^{3}$ fournit après l'essai de rupture par flexion et sciage, deux cubes $20 \times 20 \times 20 \mathrm{~cm}^{3}$. Ces cubes sont soumis à un essai de compression non confinée dont les résultats obtenus à l'âge de 28 ans, pour 4 catégories de ciment, sont récapitulés dans le tableau 3.

Les résultats présentent un écart type d'autant plus faible que la résistance à la compression est élevée.

Tableau 3: Résistances à la compression des bétons $20 \times 20 \times 20 \mathrm{~cm}^{3}$ à 28 ans

\begin{tabular}{|c|c|c|c|c|c|c|}
\hline Catégorie & Eprouvette & $\begin{array}{l}\text { Résist } \\
\text { (MPa }\end{array}$ & a compression & $\begin{array}{l}\text { Moyenne } \\
(\mathrm{MPa})\end{array}$ & Ecart & \begin{tabular}{|l} 
Ecart-type \\
$(\mathrm{MPa})$
\end{tabular} \\
\hline \multirow{3}{*}{$1:$ CPA400 } & A & 67.0 & 67.9 & \multirow{3}{*}{-65.1} & $+4.3 \%$ & \multirow{3}{*}{2.9} \\
\hline & & \begin{tabular}{|l|}
68.8 \\
--
\end{tabular} & & & & \\
\hline & $B$ & \begin{tabular}{|l|}
- \\
62.2
\end{tabular} & 62.2 & & $-4.5 \%$ & \\
\hline \multirow{3}{*}{1 bis : CPA325 } & \multirow{2}{*}{ B } & 46.3 & 437 & \multirow{3}{*}{-51.3} & $-148 \%$ & \multirow{3}{*}{8.1} \\
\hline & & 41.0 & 45.1 & & $\left.\right|^{-14.0 \%}$ & \\
\hline & C & \begin{tabular}{|l|}
56.6 \\
61.4
\end{tabular} & 59.0 & & $+15.0 \%$ & \\
\hline \multirow{4}{*}{$2:$ CPA325 } & \multirow{2}{*}{ A } & 65.2 & 641 & \multirow{4}{*}{-63.4} & $+101 \%$ & \multirow{4}{*}{1.4} \\
\hline & & 62.9 & 04.1 & & $11.1 \%$ & \\
\hline & \multirow[t]{2}{*}{$\mathrm{C}$} & 61.3 & 62.7 & & \multirow{2}{*}{$-1.1 \%$} & \\
\hline & & 64.0 & & & & \\
\hline \multirow{3}{*}{$3:$ CPA325 } & B & $\frac{48.1}{55.2}$ & 51.7 & \multirow{3}{*}{54.8} & $-5.7 \%$ & \multirow{3}{*}{4.6} \\
\hline & \multirow[b]{2}{*}{$\mathrm{C}$} & 54.4 & & & \multirow[b]{2}{*}{$+5.5 \%$} & \\
\hline & & 61.2 & 57.8 & & & \\
\hline
\end{tabular}

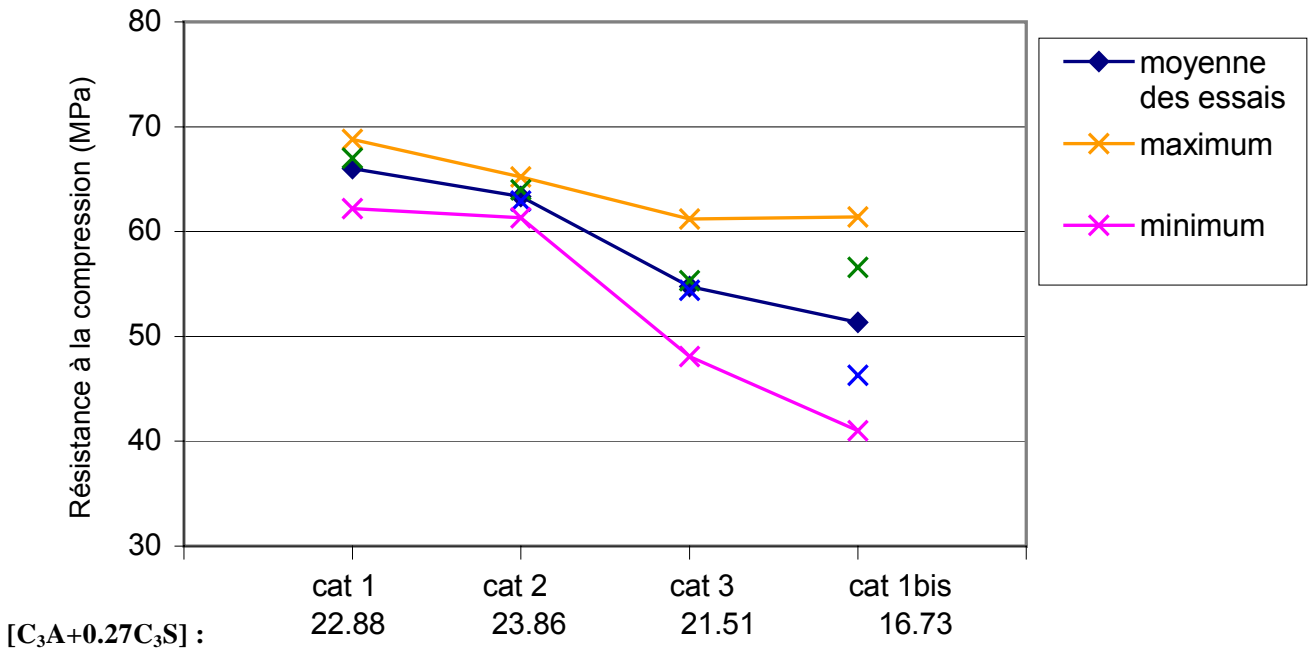

Figure3 : Evolution des écarts de résistance à la compression des bétons sur cubes à 28 ans 
Sur la figure 3 , on observe que, si la quantité $\mathrm{C}_{3} \mathrm{~A}+0.27 \mathrm{C}_{3} \mathrm{~S}$ diminue, alors la résistance moyenne décroît aussi. Le dosage en ciment de la catégorie 1 est de $400 \mathrm{~kg} / \mathrm{m}^{3}$ alors qu'il reste constant pour les catégories 1bis, 2 et 3 .

\section{Répartition des ions chlorure}

\subsection{Echantillonnage et protocole}

Après avoir effectué les essais de flexion sur les éprouvettes $20 \times 20 \times 80 \mathrm{~cm}^{3}$, on obtient deux blocs. De chaque bloc, coté opposé à la rupture, est d'abord découpé un cube $20 \times 20 \times 20 \mathrm{~cm}^{3}$. Puis du côté de la face apparente de sciage, une plaque de $2.5 \mathrm{~cm}$ d'épaisseur est découpée à sec. C'est par découpages successifs, que l'on prélève des volumes élémentaires ou échantillons de béton suivant l'axe de la plaque à espaces réguliers. Les traits de sciage sont indiqués sur la figure 4 . Ces petits éléments parallélépipédiques ainsi récupérés ont pour dimensions $4 \times 2.5 \times 1.2 \mathrm{~cm}^{3}$ excepté au centre on le volume est de $4 \times 2.5 \times 2 \mathrm{~cm}^{3}$ de taille. De ces petits éléments, on extrait en les brisant au marteau, une quantité de matrice cimentaire. Par la suite, où prépare les solutions sur les poudres de ciment et on effectue les essais de dosage des ions chlorure par la méthode de Mohr. On obtient ainsi les concentrations en ions chlorure libres et totaux.

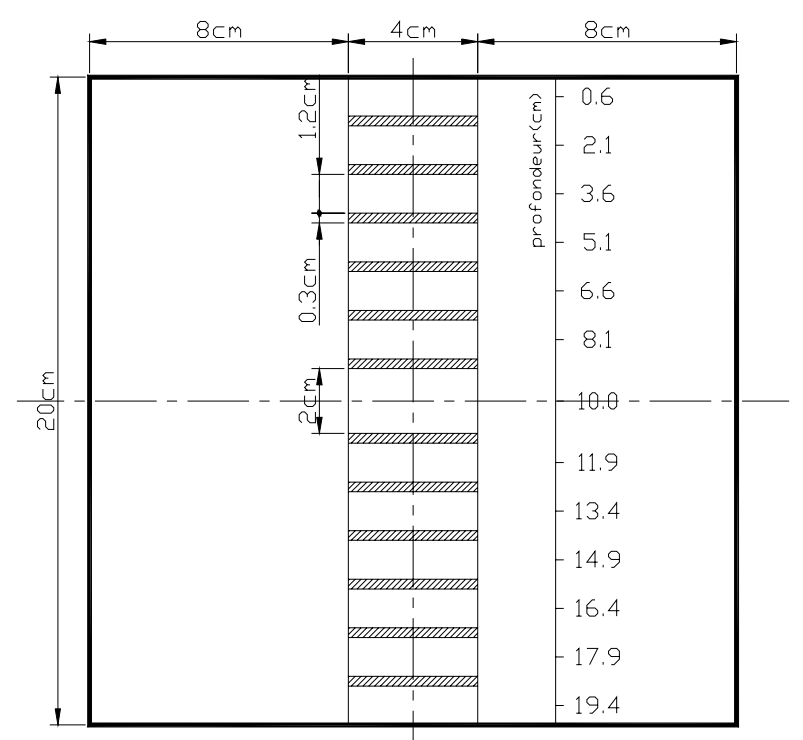

Figure 4 : Section $20 \times 20 \mathrm{~cm}^{2}$ d'éprouvette de béton découpée

Après avoir mis au point le protocole d'essai défini par Duan [4], il a été réalisé dans un premier temps des mesures sur trois éprouvettes relatives aux catégories 1, 3 et 13. Avant d'étendre les essais à la totalité des catégories, il a été prévu tout d'abord d'analyser en détails les résultats obtenus sur ces 3 catégories. Par ailleurs une autre technique de dosage des ions chlorure a été utilisée en vue de valider ces premières mesures et poursuivre les mesures sur les autres catégories, [5]. 


\subsection{Commentaires et observations pour la catégorie 1}

D'après la figure 5 , les deux courbes de pénétration des chlorures libres et totaux sont très proches, en particulier à l'intérieur de la section en béton, pour une profondeur variant de $5.1 \mathrm{~cm}$ à $14.9 \mathrm{~cm}$. Les chlorures totaux diminuent fortement de $0.72 \%$ à $0.27 \%$ entre la face externe et une profondeur de $6.6 \mathrm{~cm}$.

Ils augmentent ensuite lentement pour atteindre une valeur de $0.58 \%$ à l'approche de l'autre face. On observe clairement que la valeur obtenue à $0.6 \mathrm{~cm}$ est supérieure à la valeur mesurée à $19.4 \mathrm{~cm}$, qui se situe à la même profondeur de $0.6 \mathrm{~cm}(0.6=20-19.4)$ sur la face opposée. Cette dissymétrie peut s'expliquer. En effet, il y a une des deux faces qui est plus exposée à la mer, voir figure 1.

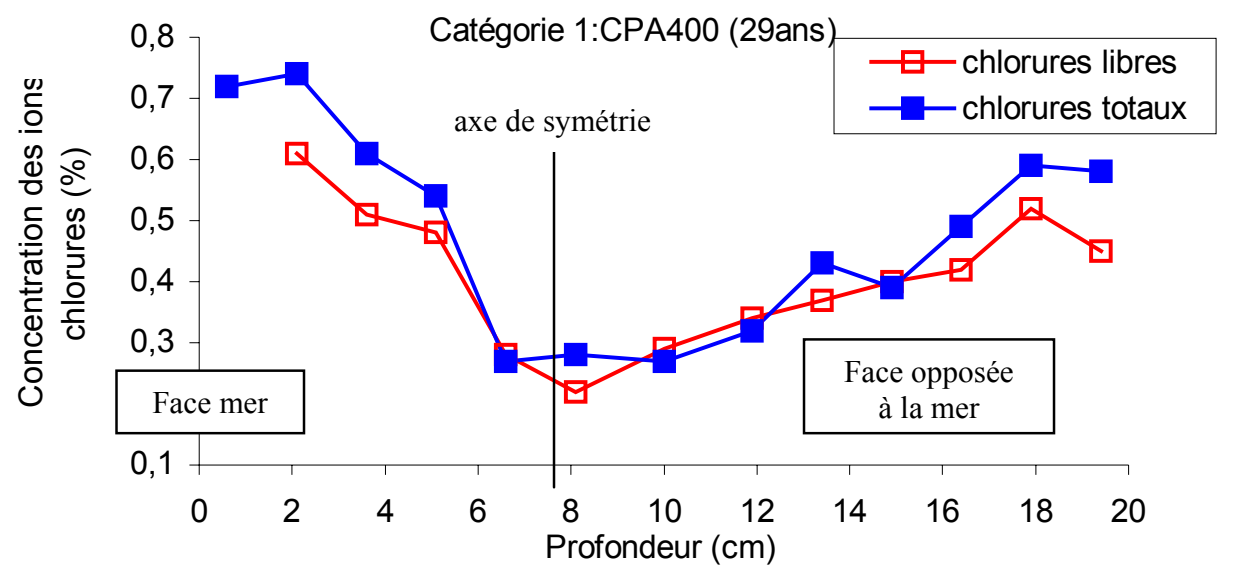

Figure 5: Concentrations des ions chlorure dans les bétons $20 \times 20 \times 80 \mathrm{~cm}^{3}$

\subsection{Commentaires et observations pour la catégorie 3}

Comparativement à la catégorie 1, on observe sur la figure 6 que les valeurs de concentration de la catégorie 3 varient plus lentement. Le taux le plus faible des chlorures libres est situé à mi-profondeur, à savoir $10 \mathrm{~cm}$, avec une concentration inférieure à $0.2 \%$ d'environ. On sait que le comportement en résistance à la compression de la catégorie 1 est plus élevé que celui de la catégorie 3 .

Cette catégorie a normalement une bonne capacité pour résister à la pénétration des chlorures, mais les résultats obtenus ne le confirment pas. La pénétration est un processus complexe de longue durée. La résistance à la compression n'est pas un bon indicateur de la pénétration des chlorures. Peut-être faut-il alors considérer les constituants du ciment utilisé? 


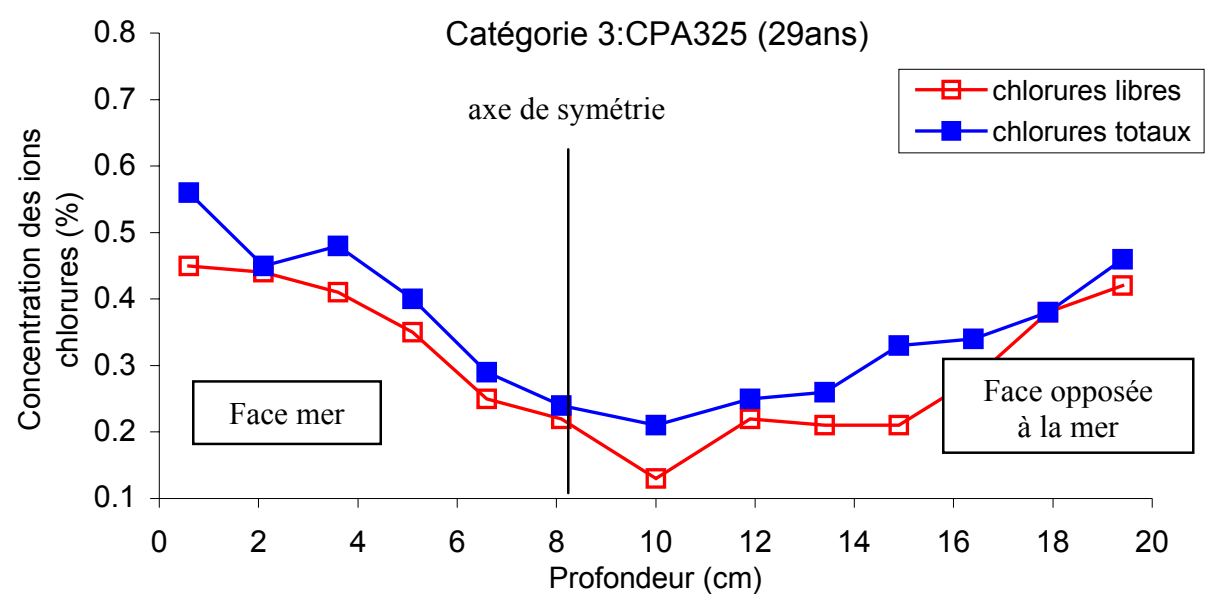

Figure 6 : Concentrations des ions chlorure dans les bétons $20 \times 20 \times 80 \mathrm{~cm}^{3}$

\subsection{Commentaires et observations pour la catégorie 13}

Rappelons que la catégorie 13 a donné lieu à des confections d'éprouvettes décalées dans le temps. Elles sont plus jeunes de 4 ans par rapport à l'âge des catégories 1 et 3 , donc les durées d'immersion ne sont pas les mêmes. La variation de la concentration en fonction de la profondeur est moins significative comme l'atteste la figure 7.

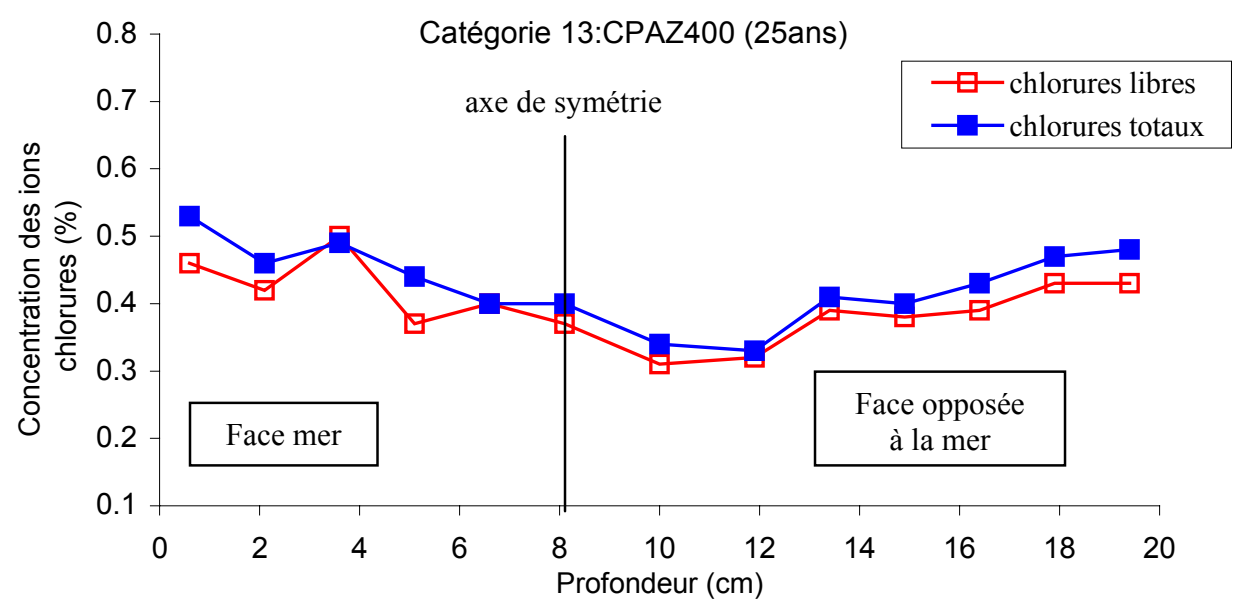

Figure 7 : Concentrations des ions chlorure dans les bétons $20 \times 20 \times 80 \mathrm{~cm}^{3}$ 


\subsection{Etude comparative des résultats à 29 ans pour les catégories 1,3 et 13}

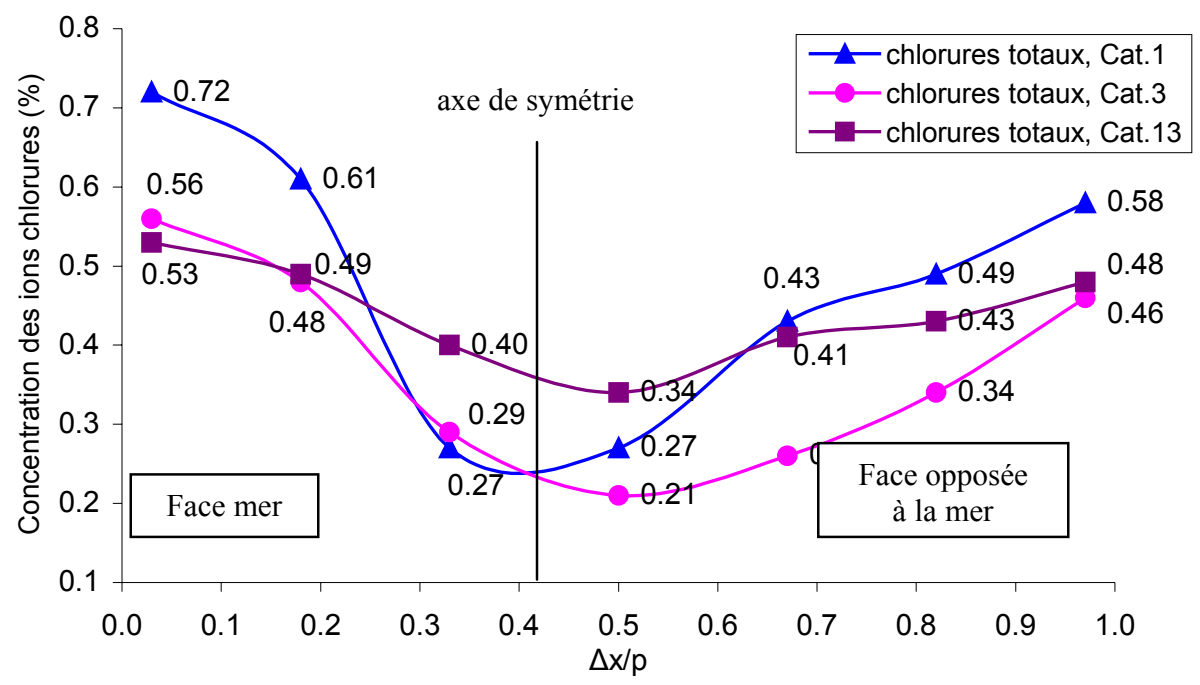

Figure 8 : Concentrations des ions chlorure totaux dans les bétons cas des catégories 1(29 ans), 3(29 ans) et 13(25 ans)

Une première analyse peut être menée sur l'ensemble des résultats pour ces trois catégories sur une période égale à 29 ans. Sur les trois courbes de concentrations respectives en fonction de la profondeur relative $\Delta \mathrm{x} / \mathrm{p}$ ( $\mathrm{p}$ indique la profondeur), présentent toute une nette dissymétrie selon l'exposition de l'éprouvette à la mer. Le tableau 4 récapitule les concentrations $\mathrm{C}_{\mathrm{i}}$ caractéristiques mesurées à savoir: $\mathrm{C}_{1}$ (coté face mer), $\mathrm{C}_{2}$ (au cœur du matériau) et $\mathrm{C}_{3}$ (face opposée); les écarts de concentration entre les faces opposées $\Delta \mathrm{C}_{1}=\mathrm{C}_{1}$ $\mathrm{C}_{3}$ et, entre la face exposée et le cœur du matériau $\Delta \mathrm{C}_{2}=\mathrm{C}_{1}-\mathrm{C}_{2}$; les rapports de concentration $\mathrm{R}_{1}=\mathrm{C}_{1} / \mathrm{C}_{3}$ et $\mathrm{R}_{2}=\mathrm{C}_{1} / \mathrm{C}_{2}$. On peut aussi évaluer un taux de pénétration en fonction du temps $\mathrm{TP}=\Delta \mathrm{C}_{2} / \mathrm{T}$, avec $\mathrm{T}$ égal à la durée d'immersion.

Tableau 4 : Différence des taux de pénétration entre les deux faces de la poutre et taux de pénétration par an

\begin{tabular}{|l|l|l|l|l|l|l|l|l|}
\cline { 2 - 9 } \multicolumn{1}{c|}{} & $\begin{array}{l}\Delta \mathrm{x} / \mathrm{p}=0.0 \\
3\end{array}$ & $\begin{array}{l}\Delta \mathrm{x} / \mathrm{p}=0.5 \\
0\end{array}$ & $\begin{array}{l}\Delta \mathrm{x} / \mathrm{p}=0.9 \\
\mathrm{C}_{1}(\%)\end{array}$ & $\begin{array}{l}\Delta \mathrm{C}_{1}(\%) \\
(\%)\end{array}$ & $\begin{array}{l}\Delta \mathrm{C}_{2} \\
(\%)\end{array}$ & $\mathrm{R}_{1}$ & $\mathrm{R}_{2}$ & $\begin{array}{l}\mathrm{TP} \\
(\%) \\
\text { an })\end{array}$ \\
\hline Cat. 1 & 0.72 & 0.27 & 0.58 & 0.14 & 0.45 & 1.24 & 2.67 & 0.016 \\
\hline Cat. 3 & 0.56 & 0.21 & 0.48 & 0.08 & 0.35 & 1.17 & 2.67 & 0.012 \\
\hline $\begin{array}{l}\text { Cat. } \\
13\end{array}$ & 0.53 & 0.34 & 0.46 & 0.07 & 0.19 & 1.15 & 1.56 & 0.008 \\
\hline
\end{tabular}

On note qu'il y a des différences notables entre les deux côtés (face et dos à la mer), cette différence varie de 0.07 à 0.14 selon la catégorie. Parmi ces bétons observés, le comportement de la catégorie 1 s'avère être le plus mauvais. Pour la catégorie 13 (CPAZ 400), le taux de pénétration est le plus faible par an. Il faut tenir compte de la variabilité des prélèvements d'échantillons à long terme et à leur période d'immersion de 29 ans et 25 ans. Un seul échantillon ne peut pas représenter complètement le comportement réel. 


\section{Conclusions}

Il est nécessaire de connaître la composition chimique du ciment, si l'on s'intéresse à la durabilité des bétons à la mer. En effet, certains composés principalement le $\mathrm{C}_{3} \mathrm{~A}, \mathrm{SO}_{3}$ et aussi le $\mathrm{C}_{3} \mathrm{~S}$, peuvent réagir avec des éléments contenus dans l'eau de mer. De ces essais nous pouvons constater les phénomènes suivants:

- la quantité $\mathrm{C}_{3} \mathrm{~A}+0.27 \mathrm{SO}_{3}$ joue un rôle important dans la résistance en compression à long terme des bétons immergés, pour des ciments CPA325.

- la pénétration des ions chlorure se manifeste differemment selon l'exposition de la face à la mer, Cependant la pénétration des chlorures dans les bétons est un processus complexe qui se prolonge dans le temps. Il est cependant intéressant de déterminer une relation entre les concentrations des ions chlorure et la profondeur, comme une relation entre la vitesse de pénétration et le temps.

\section{Références}

1.BOUTOUIL M., CAMINADE D., HENRY D., LEVACHER D., (1999). Durabilité des mortiers et des bétons en milieu marin naturel e reconstitué-comportement mécanique et chimique à long term. Rapport de contrat, $\mathrm{n}^{\circ} 97 / \mathrm{POS} / \mathrm{STC} / \mathrm{ATILH}$. Université de Nantes, $176 \mathrm{p}$.

2.BOUTOUIL M.,(1995). Comportement d'éprouvettes de mortiers et de bétons à la mer, Rapport de DEA Génie Civil, Université de Nantes, 41-75.

3.DREUX G., (1970). Guide pratique du béton, Ed. Eyrolles, 257 p.

4.DUAN Z., (2003). Durabilité des mortiers et bétons à la mer - Pénétration des chlorures, Rapport de DEA Génie Côtier, Université de Caen, 35-43.

5.TANG Z., (2004). Etude du comportement et de la durabilité des bétons à la mer, Rapport de DEA, Université de Caen, 49-63. 